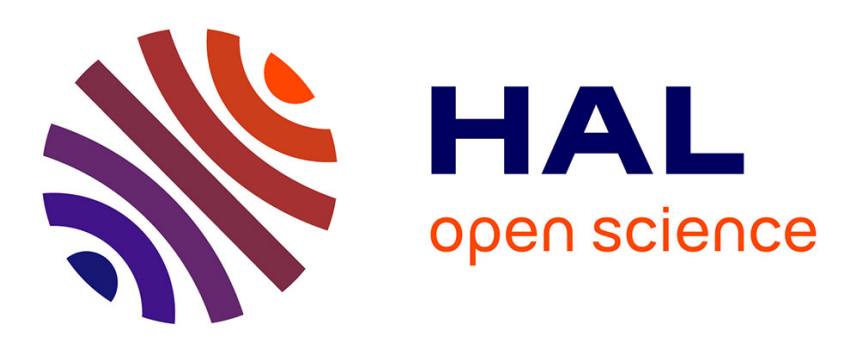

\title{
Quantifying the Uncertainties-Induced Errors in Robot Impact Detection Methods
}

Nolwenn Briquet-Kerestedjian, Maria Makarov, Pedro Rodriguez-Ayerbe, Mathieu Grossard

\section{- To cite this version:}

Nolwenn Briquet-Kerestedjian, Maria Makarov, Pedro Rodriguez-Ayerbe, Mathieu Grossard. Quantifying the Uncertainties-Induced Errors in Robot Impact Detection Methods. IECON 2016 - 42nd Annual Conference of IEEE Industrial Electronics Society, Oct 2016, Florence, Italy. pp.5328-5334, 10.1109/iecon.2016.7793186 . hal-01416124

HAL Id: hal-01416124

https://hal-centralesupelec.archives-ouvertes.fr/hal-01416124

Submitted on 14 Dec 2016

HAL is a multi-disciplinary open access archive for the deposit and dissemination of scientific research documents, whether they are published or not. The documents may come from teaching and research institutions in France or abroad, or from public or private research centers.
L'archive ouverte pluridisciplinaire HAL, est destinée au dépôt et à la diffusion de documents scientifiques de niveau recherche, publiés ou non, émanant des établissements d'enseignement et de recherche français ou étrangers, des laboratoires publics ou privés. 


\section{Quantifying the Uncertainties-Induced Errors in Robot Impact Detection Methods}

\author{
N. Briquet-Kerestedjian, M. Makarov, P. Rodriguez-Ayerbe \\ Laboratoire des Signaux et Systèmes (L2S), \\ CentraleSupélec-CNRS-Univ. Paris-Sud, Université Paris-Saclay, \\ F91192, Gif-sur-Yvette, France \\ Email: nolwenn.briquet-kerestedjian@centralesupelec.fr
}

\author{
N. Briquet-Kerestedjian, M. Grossard \\ CEA, LIST, \\ Interactive Robotics Laboratory, \\ F-91191 Gif-sur-Yvette, France \\ Email: mathieu.grossard@cea.fr
}

\begin{abstract}
In the context of human-robot collaboration, an efficient impact detection is essential for safe operation. Residualbased collision detection relies on the difference between the estimated and actual motor torques. However, in these modelbased methods uncertainties affect the residual in the same structural way as a collision does, leading to potential false alarms. This paper proposes to quantify the influence of uncertainties on residual generation methods based on the inverse dynamic model for both rigid and elastic-joint robots. These uncertainties-induced errors are investigated depending on their origin (parameters estimation or numerical differentiation). Boundaries of these errors are determined along a given trajectory and account as the minimum threshold of detectability of a collision. These results are illustrated in simulation.
\end{abstract}

\section{INTRODUCTION}

In industrial or service applications, human-robot interaction to perform tasks in cooperation involves sharing the same workspace. In this context, appropriate regulations and technologies must ensure safety and minimize the risk of injury for the operator in case of collision with the robot [1], [2].

To prevent a collision, exteroceptive sensors and supervisions systems (such as computer vision [3], 3D-simulation, etc.) can be used to detect any unexpected presence in the robot area and adapt its trajectory accordingly. In case of failure of these techniques (e.g. obstruction of the field of vision or breakdown) or to furthermore enhance security, a certain number of methods are proposed to detect rapidly, sensitively and reliably a collision. To obtain a fast detection, robot capabilities can be extended by the use of additional sensors such as skin sensors [4] or proximity and contact force sensors [5], however often at the expense of cost and integration constraints. Other collision detection approaches use only proprioceptive sensors, e.g. motor and joint position sensors or joint torque sensors [6]. Their reliability, essential for the operator safety, is directly related to the sensitivity of the selected impact detection method.

Collision detection approaches are often derived from fault diagnostic methods, since collisions can be viewed as faulty behaviours that introduce changes in the state variables or parameters of a static or dynamic system as described in [7]. The diagnostic is composed of two steps : the residual generation followed by its evaluation. A classical residual generation method for collision detection is based on the difference between the actual applied motor torque and the estimated motor torque obtained from the robot dynamic model in the absence of any external force. To estimate the motor torque, a basic approach uses the inverse dynamic model based on the estimated parameters and configuration coordinates without any external disturbance as in [8] and [9]. Another widely used method relies on the generalized momentum of the robot [10], [11], [12]. This method has the advantage of avoiding the measure or computation of motor and joint accelerations that generate measurement noise and delay. As part of this study, only the basic method based on the inverse dynamic model of the robot will be studied at first.

These residual generation approaches are model-based methods which are affected by uncertainties if the model is not exactly known or is not properly adapted to the operational conditions (e.g. varying payload or temperature). Consequently, the estimated motor torque contains errors which affect the residual generation in the same structural way as an external force. Several approaches propose to account for uncertainties in the detection method. A Kalman filter with friction uncertainties is designed in [12] to increase the robustness of the generalized momentum observer method. For the same method, an adaptive fault and detection isolation scheme has been proposed in [13] to deal with parametric uncertainties. In a similar perspective, a new residual evaluation approach has been developed in [14] based on a dynamic statedependent threshold and an online estimated filter to adapt the algorithm to different operating conditions but without dealing explicitly with uncertainties.

This paper proposes to quantify the influence of uncertainties on the residual for both rigid and elastic-joint backdrivable robots with only motor and joint positions sensors. Indeed, despite the absence of force/torque sensor, robots with good backdrivable properties can achieve a very sensitive collision detection using only motor-side measurements, as in [14], since external forces are accurately reflected on motor shafts. This approach is derived for basic residual generation methods using the inverse dynamic model of the robot. Uncertainties are expressed as a contribution of both parameters estimation and numerical differentiation errors. The total uncertaintiesinduced errors are investigated and boundaries are determined along the trajectory, which allows to quantify the minimum 
threshold of detectability of a collision, that is the sensitivity of the collision detection method. Simulation results on a 6 degrees-of-freedom (DOF) manipulator robot highlight the influence of each type of uncertainties on the residual depending on the trajectory and illustrate the level of sensitivity of the method. These results give an estimate of the uncertaintiesinduced errors that can be expected online and allow to choose the method that will generate the least errors on the detection. The proposed approach can be extended to other model-based detection methods.

Section II recalls the inverse dynamic model of both rigid and elastic-joint serial robot manipulators and investigates the potential sources of uncertainties. In Section III, after a description of the problem of residual generation in presence of uncertainties, induced errors are derived using the inverse dynamic model for both rigid and elastic-joint cases and boundaries on each type of uncertainties are proposed. Section IV is devoted to the presentation of the simulation results.

Mathematical notations: In the following, $\stackrel{\text { def }}{=}$ means "by definition", $a_{\star}$ denotes the measured or estimated value of any matrix or vector variable $\boldsymbol{a}$, and $\boldsymbol{\delta} \boldsymbol{a}$ is defined as the difference between the measured or estimated value $a_{\star}$ and the exact value $a . z$ and $s$ denote respectively the complex $\mathrm{z}$ domain variable and the Laplace-domain variable. In discrete time, $T_{s}$ is the sampling time. The notation $\boldsymbol{a} \sim \mathcal{N}\left(\boldsymbol{\mu}, \boldsymbol{\sigma}^{2}\right)$ means that $\boldsymbol{a}$ follows a normal distribution with mean $\boldsymbol{\mu}$ and variance $\sigma^{2}$.

\section{MODEL DESCRIPTION}

For serial robot manipulators which dynamic models are briefly recalled, uncertainties may be at least of two types: parametric uncertainties due to an imperfect knowledge of the robot parameters and uncertainties due to numerical differentiation with respect to time (speed and acceleration estimations).

\section{A. Dynamic model for serial robot manipulators}

The dynamic behaviour of a serial robot manipulator with $n$-rigid links and joints can be described by its inverse dynamic model [15] as follows

$$
\boldsymbol{M}_{\text {rig }}(\boldsymbol{q}) \ddot{\boldsymbol{q}}+\boldsymbol{C}(\boldsymbol{q}, \dot{\boldsymbol{q}}) \dot{\boldsymbol{q}}+\boldsymbol{G}(\boldsymbol{q})+\boldsymbol{\tau}_{f}(\dot{\boldsymbol{q}})=\boldsymbol{\tau}_{m}+\boldsymbol{\tau}_{\text {ext }} \stackrel{\text { def }}{=} \boldsymbol{\tau}
$$

where $\boldsymbol{q} \in \mathbb{R}^{n}$ is the joint position vector, $\boldsymbol{M}_{\text {rig }}(\boldsymbol{q})=$ $\boldsymbol{M}(\boldsymbol{q})+\boldsymbol{J}_{m o t}$ where $\boldsymbol{M} \in \mathbb{R}^{n \times n}$ is the robot inertia matrix and $\boldsymbol{J}_{m o t} \in \mathbb{R}^{n \times n}$ is the constant diagonal motor inertia matrix after the reduction stage, $\boldsymbol{C}(\boldsymbol{q}, \dot{\boldsymbol{q}}) \dot{\boldsymbol{q}} \in \mathbb{R}^{n}$ captures the Coriolis and centrifugal torques, $\boldsymbol{G}(\boldsymbol{q}) \in \mathbb{R}^{n}$ represents the contribution of the gravity torque, $\boldsymbol{\tau}_{f}(\dot{\boldsymbol{q}}) \in \mathbb{R}^{n}$ is the friction torque which is expressed by $\boldsymbol{\tau}_{f}(\dot{\boldsymbol{q}})=\boldsymbol{F}_{v} \dot{\boldsymbol{q}}+\boldsymbol{F}_{s} \operatorname{sign}(\dot{\boldsymbol{q}})$ in case of viscous and Coulomb friction with $\boldsymbol{F}_{v}$ and $\boldsymbol{F}_{s}$ respectively the viscous and Coulomb friction coefficients, $\boldsymbol{\tau}_{m} \in \mathbb{R}^{n}$ denotes the applied motor torque after the reduction stage and $\tau_{\text {ext }} \in \mathbb{R}^{n}$ expresses the external torque vector. $\boldsymbol{\tau}$ is the global motion torque. For DC motors, the motor torque $\tau_{m}$ is related to the motor currents $\boldsymbol{i}_{m}$ by $\boldsymbol{\tau}_{m}=\boldsymbol{R}_{r e d}^{T} \boldsymbol{K}_{e m} \boldsymbol{i}_{m}$ with $\boldsymbol{R}_{r e d}$ the reduction matrix and $\boldsymbol{K}_{e m}$ the diagonal matrix of torque constants of the involved motors. In case of backdrivable robots, the external forces are accurately reflected on the motor shafts, which allows their detection without any additional joint torque sensor. If an external force $\boldsymbol{F}_{\text {ext }} \in \mathbb{R}^{n}$ collides with the $i^{t h}$ link of the robot, then $\boldsymbol{F}_{\text {ext }}$ is related to $\boldsymbol{\tau}_{\text {ext }}$ by $\boldsymbol{\tau}_{\text {ext }}=\boldsymbol{J}^{T}(\boldsymbol{q}) \boldsymbol{F}_{\text {ext }}$ where $\boldsymbol{J}(\boldsymbol{q})$ is the Jacobian matrix of the $i^{\text {th }}$ link.

For the purpose of human-robot collaboration, lightweight robot manipulators can be suitable because of their lower inertia which reduces the maximal torque in case of impact. The elastic-joint model (2-3) accounts for the elasticity of the transmissions :

$$
\begin{aligned}
\boldsymbol{M}(\boldsymbol{q}) \ddot{\boldsymbol{q}}+\boldsymbol{C}(\boldsymbol{q}, \dot{\boldsymbol{q}}) \dot{\boldsymbol{q}}+\boldsymbol{G}(\boldsymbol{q})+\boldsymbol{\tau}_{f a}(\dot{\boldsymbol{q}})+\boldsymbol{K}(\boldsymbol{q}-\boldsymbol{\theta}) & =\boldsymbol{\tau}_{e x t} \\
\boldsymbol{J}_{m o t} \ddot{\boldsymbol{\theta}}+\boldsymbol{\tau}_{f m}(\dot{\boldsymbol{\theta}})-\boldsymbol{K}(\boldsymbol{q}-\boldsymbol{\theta}) & =\boldsymbol{\tau}_{m}
\end{aligned}
$$

where $\boldsymbol{\theta} \in \mathbb{R}^{n}$ is the motor position vector after the reduction stage, $\boldsymbol{K} \in \mathbb{R}^{n \times n}$ is the stiffness matrix assumed to be constant and diagonal in this study, $\boldsymbol{\tau}_{f a}(\dot{\boldsymbol{q}}) \in \mathbb{R}^{n}$ and $\boldsymbol{\tau}_{f m}(\dot{\boldsymbol{\theta}}) \in \mathbb{R}^{n}$ are respectively the joint and motor friction torques which are, considering viscous and Coulomb friction, $\boldsymbol{\tau}_{f a}(\dot{\boldsymbol{q}})=\boldsymbol{F}_{v a} \dot{\boldsymbol{q}}+\boldsymbol{F}_{s a} \operatorname{sign}(\dot{\boldsymbol{q}})$ at joint level and $\boldsymbol{\tau}_{f m}(\dot{\boldsymbol{\theta}})=\boldsymbol{F}_{v m} \dot{\boldsymbol{\theta}}+\boldsymbol{F}_{s m} \operatorname{sign}(\dot{\boldsymbol{\theta}})$ at motor level, with $\left(\boldsymbol{F}_{v a}, \boldsymbol{F}_{v m}\right)$ the viscous friction coefficients and $\left(\boldsymbol{F}_{s a}, \boldsymbol{F}_{s m}\right)$ the Coulomb friction coefficients. Summing (2) and (3) it follows

$$
\begin{aligned}
\boldsymbol{M}(\boldsymbol{q}) \ddot{\boldsymbol{q}} & +\boldsymbol{C}(\boldsymbol{q}, \dot{\boldsymbol{q}}) \dot{\boldsymbol{q}}+\boldsymbol{G}(\boldsymbol{q})+\boldsymbol{\tau}_{f a}(\dot{\boldsymbol{q}})+\boldsymbol{J}_{m o t} \ddot{\boldsymbol{\theta}}+\boldsymbol{\tau}_{f m}(\dot{\boldsymbol{\theta}}) \\
& =\boldsymbol{\tau}_{m}+\boldsymbol{\tau}_{\text {ext }} \stackrel{\text { def }}{=} \boldsymbol{\tau}
\end{aligned}
$$

Let us note that when the robot is considered as completely rigid $(\boldsymbol{K} \rightarrow \infty), \boldsymbol{\theta}=\boldsymbol{q}$ and we obtain (1).

\section{B. Model uncertainties}

For model-based approaches, robot dynamic parameters can be obtained from the nominal CAD data or identified experimentally as they can slightly differ from one robot to another or for different operating conditions. Their experimental identification [15] uses the fact that the inverse dynamic model (1) of an $n$-degrees of freedom rigid serial robot can be written as a linear regression with respect to the $n_{b}$ rigid base parameters

$$
\boldsymbol{\tau}_{m}=\boldsymbol{\varphi}_{\text {rig }}(\boldsymbol{q}, \dot{\boldsymbol{q}}, \ddot{\boldsymbol{q}}) \chi_{\text {rig }}
$$

where $\varphi_{\text {rig }} \in \mathbb{R}^{n \times n_{b}}$ is the rigid regression matrix and $\chi_{\text {rig }} \in$ $\mathbb{R}^{n_{b}}$ is the vector of rigid base parameters (i.e. the minimal set of identifiable parameters). For each link, the latter are obtained by linear combinations of the 6 components of the inertia tensor, the 3 components of the first moment and the mass, the total inertia moment for rotor actuator and gears, and the viscous and Coulomb friction coefficients. For an elasticjoint robot, the linear regression can be written with respect to the $n_{b}+n_{f}$ rigid and flexible base parameters [16] as follows

$$
\left(\begin{array}{c}
0_{n \times n} \\
\boldsymbol{\tau}_{m}
\end{array}\right)=\boldsymbol{\varphi}_{f l}(\boldsymbol{q}, \dot{\boldsymbol{q}}, \ddot{\boldsymbol{q}}, \boldsymbol{\theta}, \dot{\boldsymbol{\theta}}, \ddot{\boldsymbol{\theta}}) \chi_{f l}
$$

where $\varphi_{f l} \in \mathbb{R}^{2 n \times\left(n_{b}+n_{f}\right)}$ is the flexible regression matrix and $\chi_{f l} \in \mathbb{R}^{n_{b}+n_{f}}$ is the vector of the rigid and flexible base parameters. It is composed of the base parameters distinguishing rigid and flexible parameters, such as viscous and Coulomb 
friction at motor and joint levels, and including the stiffness parameters.

After evaluating the identification model (5) or (6) respectively in the rigid or elastic-joint case at a sufficient number of points on several exciting trajectories, the vector $\chi_{\star}$ of the identified base parameters can be obtained by least squares minimization of the 2-norm of the residual errors vector. We denote $\delta \chi$ the vector of estimation errors defined by

$$
\delta \chi \stackrel{\text { def }}{=} \chi_{\star}-\chi
$$

For the following, we assume that $\delta \chi$ is Gaussian with zeromean and standard deviation $\sigma_{\chi_{\star}}$ which can be obtained from the estimation of the standard deviation of the errors vector resulting from the identification. The relative standard deviation $\% \sigma_{\chi_{\star, i}}$ of the $i^{t h}$ identified parameter is defined as

$$
\% \sigma_{\chi_{\star, i}}=100 \frac{\sigma_{\chi_{\star, i}}}{\left|\chi_{\star, i}\right|}
$$

$\% \sigma_{\chi_{\star, i}}$ is used as a criterion to measure the quality of the identification of the $i^{t h}$ base parameter. Generally, we consider that the identification is acceptable if the relative standard deviation of a parameter is less than ten percent.

The resulting model errors are obtained by difference between the matrices evaluated with the identified parameters and with the exact parameters. For example for the inertia matrix,

$$
\boldsymbol{\delta} \boldsymbol{M}_{\text {rig }}(\boldsymbol{q}) \stackrel{\text { def }}{=} \boldsymbol{M}_{\text {rig }}(\boldsymbol{q})-\boldsymbol{M}_{\text {rig }}(\boldsymbol{q})
$$

with $\boldsymbol{M}_{r i g \star}$ and $\boldsymbol{M}_{\text {rig }}$ denoting the inertia matrix evaluated respectively with the identified parameters and with the exact parameters. Calculating the difference between the inverse dynamic model evaluated with the identified and the exact parameters, an equivalent linear regression with respect to the estimation errors is obtained. In the rigid case, we obtain

$$
\begin{aligned}
\delta \boldsymbol{M}_{r i g}(\boldsymbol{q}) \ddot{\boldsymbol{q}} & +\boldsymbol{\delta} \boldsymbol{C}(\boldsymbol{q}, \dot{\boldsymbol{q}}) \dot{\boldsymbol{q}}+\boldsymbol{\delta} \boldsymbol{G}(\boldsymbol{q})+\boldsymbol{\delta} \boldsymbol{F}_{v} \dot{\boldsymbol{q}}+\boldsymbol{\delta} \boldsymbol{F}_{s} \operatorname{sign}(\dot{\boldsymbol{q}}) \\
& =\boldsymbol{\varphi}_{\text {rig }}(\boldsymbol{q}, \dot{\boldsymbol{q}}, \ddot{\boldsymbol{q}}) \boldsymbol{\delta} \boldsymbol{\chi}_{r i g}
\end{aligned}
$$

\section{Numerical differentiation errors}

Another origin of the errors comes from the numerical differentiation. Indeed, when only position sensors are integrated into the robot, in an industrial context velocities and accelerations are often rather approximated by finite differences from Taylor series than estimated by observers. For this study, we will consider the example of two common numerical differentiation methods and study the corresponding errors.

- Method $D_{1}$ : Fourth-order backward finite difference

$$
G_{1}(z)=\frac{2+z^{-1}-z^{-3}-2 z^{-4}}{10 T_{s}} .
$$

- Method $\boldsymbol{D}_{2}$ : First-order low-pass filtered derivative

$$
G_{2}(z)=\alpha \frac{1-z^{-1}}{1-\beta z^{-1}}
$$

with $\alpha=\left(d \omega_{c}\right) /(1+d), \beta=(d-1) /(d+1)$ and $d=$ $2 /\left(\omega_{c} T_{s}\right), \omega_{c}$ being the cut-off frequency.

We denote $H_{1}(s)$ and $H_{2}(s)$ the equivalent continuous filters obtained by bilinear transform of respectively $G_{1}(z)$ and

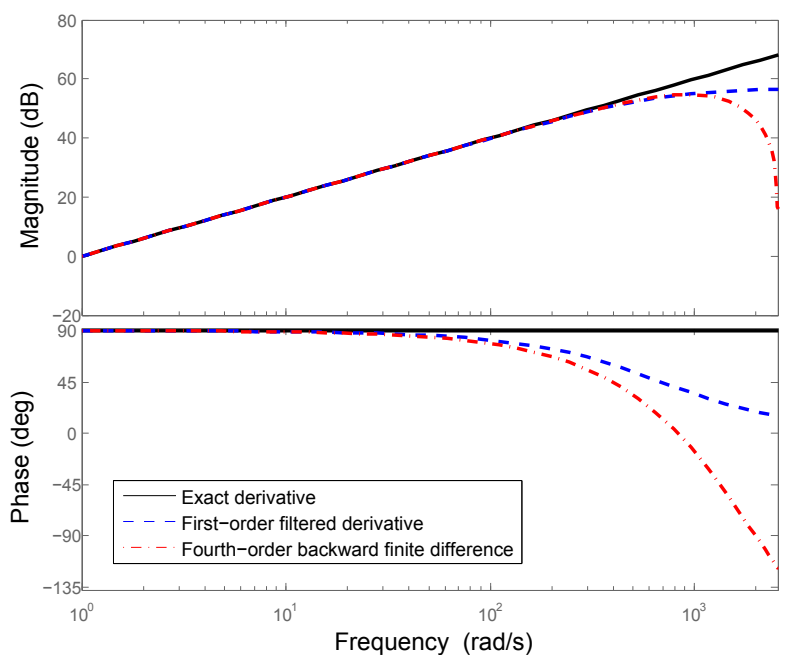

Fig. 1: Frequency responses of continuous exact derivative, first-order backward finite difference and first-order filtered derivative methods with $\omega_{c}=2 \pi 110 \mathrm{rad} / \mathrm{s}$ and $T_{s}=1 \mathrm{~ms}$

$G_{2}(z)$. Fig. 1 compares the two numerical derivation scheme in the frequency domain. For both approaches, the approximation is significantly deteriorated at high frequencies since the signal is low-pass filtered and the phase shifted.

More generally, regardless of the chosen numerical differentiation filter, when derivatives are estimated from noisy measurements, the error with respect to the exact derivative of the signal is twofold: it contains an error term due to the derivative approximation, and an error term due to filtered measurement noise. We denote $\boldsymbol{q}_{\star}$ the measured joint positions and define $\dot{\boldsymbol{q}}_{\star}$ and $\ddot{\boldsymbol{q}}_{\star}$ respectively the joint velocities and accelerations obtained by numerical differentiation of $\boldsymbol{q}_{\star}$ (and similarly for the measured motor positions $\boldsymbol{\theta}_{\star}$ ). We also define $\delta \dot{\boldsymbol{q}}$ the vector of errors due to numerical differentiation of joint velocities as

$$
\delta \dot{\boldsymbol{q}} \stackrel{\text { def }}{=} \dot{\boldsymbol{q}}_{\star}-\dot{\boldsymbol{q}}
$$

Similarly we obtain $\delta \ddot{q}, \delta \dot{\boldsymbol{\theta}}$ and $\boldsymbol{\delta} \ddot{\boldsymbol{\theta}}$ respectively for the joint accelerations and the motor velocities and accelerations.

Let $\boldsymbol{q}_{\star}=\boldsymbol{q}+\boldsymbol{\xi}$ be the noisy position measurement affected by a white bounded noise $\xi$ due for instance to quantization. In the Laplace-domain, the error $\delta \dot{q}_{j}$ due to numerical differentiation on the $j^{t h}$ axis velocity is given by

$$
\Delta Q_{d}^{j}(s)=\underbrace{[H(s)-s] Q^{j}(s)}_{\text {approximated velocity-induced error }}+\underbrace{H(s) \xi^{j}(s)}_{\text {filtered noise }}
$$

where $\Delta Q_{d}^{j}(s), Q^{j}(s)$ and $\xi^{j}(s)$ are respectively the Laplace transforms of $\delta \dot{q}_{j}, q_{j}$ and $\xi_{j}$ for the $j^{t h}$ axis. Similarly we obtain the error $\delta \ddot{q}_{j}$ on the acceleration of axis $j$ in the Laplace domain

$$
\Delta Q_{d d}^{j}(s)=\left[H^{2}(s)-s^{2}\right] Q^{j}(s)+H^{2}(s) \xi^{j}(s)
$$

with $\Delta Q_{d d}^{j}(s)$ the Laplace transform of $\delta \ddot{q}_{j}$ for the $j^{t h}$ axis. We obtain similar expressions for $\boldsymbol{\delta} \dot{\boldsymbol{\theta}}$ and $\boldsymbol{\delta} \ddot{\boldsymbol{\theta}}$.

From Equations (14) and (15) the errors due to derivative approximation and the errors due to the filtered noise can be quantified and bounded (see Section III-C). 


\section{QUANTIFICATION OF THE INFLUENCE OF UNCERTAINTIES ON THE RESIDUAL}

\section{A. Problem statement}

For collision detection, a basic residual generation method can be based on the difference between the estimated global motion torque $\tau_{\star}$ and the applied motor torque $\tau_{m}$

$$
\boldsymbol{r} \stackrel{\text { def }}{=} \boldsymbol{\tau}_{\star}-\boldsymbol{\tau}_{m}
$$

The estimated global motion torque $\tau_{\star}$ is obtained from the inverse dynamic model with the matrices evaluated with the estimated parameters and coordinates, that is using (1) in the rigid case

$\boldsymbol{\tau}_{\star}=\boldsymbol{M}_{r i g \star}\left(\boldsymbol{q}_{\star}\right) \ddot{\boldsymbol{q}}_{\star}+\boldsymbol{C}_{\star}\left(\boldsymbol{q}_{\star}, \dot{\boldsymbol{q}}_{\star}\right) \dot{\boldsymbol{q}}_{\star}+\boldsymbol{G}_{\star}\left(\boldsymbol{q}_{\star}\right)+\boldsymbol{F}_{v \star} \dot{\boldsymbol{q}}_{\star}+\boldsymbol{F}_{s \star} \operatorname{sign}\left(\dot{\boldsymbol{q}}_{\star}\right)$

For the elastic-joint case we can use either (2-3) or (4) to estimate the global motion torque $\tau_{\star}$.

In the ideal case when the estimated parameters and coordinates are equal to the exact values, then

$$
\boldsymbol{\tau}_{\star} \underset{\text { ideal }}{=} \boldsymbol{\tau}=\boldsymbol{\tau}_{m}+\boldsymbol{\tau}_{\text {ext }} \Rightarrow \boldsymbol{r} \underset{\text { ideal }}{=} \boldsymbol{\tau}_{\text {ext }}
$$

However in the general case the inverse dynamic model (17) contains errors denoted $\delta \tau$ such that

$$
\tau_{\star}=\boldsymbol{\tau}+\boldsymbol{\delta} \boldsymbol{\tau} \Rightarrow \boldsymbol{r}=\boldsymbol{\tau}_{\text {ext }}+\boldsymbol{\delta} \boldsymbol{\tau}
$$

Therefore in the general case (19), even in the absence of external torque the residual is non-zero due to the presence of uncertainties that depend on the robot's state and affect the residual in the same structural way as a collision. Thus bounding the errors due to the uncertainties along the trajectory allows to predict in simulation the minimum threshold of detectability of a collision. Then an adaptive detection threshold can be set equal or above this level in order to avoid false alarms due to uncertainties.

\section{B. Influence of the uncertainties on the residual}

In this section, we give an explicit formulation of the term $\delta \boldsymbol{\tau}$ in (19) that represents all the errors induced by the uncertainties. For both rigid and elastic-joint cases, it is given by the difference between the estimated and the exact global motion torques. For the sake of simplicity, Coulomb frictions will be neglected here.

For the rigid case, we obtain from (1) and (19)

$$
\begin{aligned}
\boldsymbol{\delta} \boldsymbol{\tau}_{r i g} & =\left[\boldsymbol{M}_{r i g \star}\left(\boldsymbol{q}_{\star}\right) \ddot{\boldsymbol{q}}_{\star}+\boldsymbol{C}_{\star}\left(\boldsymbol{q}_{\star}, \dot{\boldsymbol{q}}_{\star}\right) \dot{\boldsymbol{q}}_{\star}+\boldsymbol{G}_{\star}\left(\boldsymbol{q}_{\star}\right)+\boldsymbol{F}_{v \star} \dot{\boldsymbol{q}}_{\star}\right] \\
& -\left[\boldsymbol{M}_{\text {rig }}(\boldsymbol{q}) \ddot{\boldsymbol{q}}+\boldsymbol{C}(\boldsymbol{q}, \dot{\boldsymbol{q}}) \dot{\boldsymbol{q}}+\boldsymbol{G}(\boldsymbol{q})+\boldsymbol{F}_{v} \dot{\boldsymbol{q}}\right]
\end{aligned}
$$

In (20) the exact matrices will be expressed by the difference between the matrices evaluated with the identified parameters and their error estimation term using (9). It is assumed that $\boldsymbol{M}_{r i g \star}\left(\boldsymbol{q}_{\star}\right) \approx \boldsymbol{M}_{r i g \star}(\boldsymbol{q}), \boldsymbol{C}_{\star}\left(\boldsymbol{q}_{\star}, \dot{\boldsymbol{q}}_{\star}\right) \approx \boldsymbol{C}_{\star}(\boldsymbol{q}, \dot{\boldsymbol{q}})$ and $\boldsymbol{G}_{\star}\left(\boldsymbol{q}_{\star}\right) \approx \boldsymbol{G}_{\star}(\boldsymbol{q})$ since small variations in $\boldsymbol{q}$ or $\dot{\boldsymbol{q}}$ induce small variations in the trigonometric functions of $M_{r i g \star}, C_{\star}$ and $G_{\star}$. Thus the error terms due to numerical differentiation appear using (13). We finally obtain the theoretical expression of $\delta \tau$ for the rigid case

$$
\boldsymbol{\delta} \tau_{\text {rig }}=\boldsymbol{\delta} \tau_{\text {rig }}^{p}+\boldsymbol{\delta} \tau_{\text {rig }}^{d}
$$

with

$$
\begin{aligned}
& \boldsymbol{\delta} \tau_{r i g}^{p}=\boldsymbol{\delta} \boldsymbol{M}_{r i g}(\boldsymbol{q}) \ddot{\boldsymbol{q}}+\boldsymbol{\delta} \boldsymbol{C}(\boldsymbol{q}, \dot{\boldsymbol{q}}) \dot{\boldsymbol{q}}+\boldsymbol{\delta} \boldsymbol{G}(\boldsymbol{q})+\boldsymbol{\delta} \boldsymbol{F}_{v} \dot{\boldsymbol{q}} \\
& \boldsymbol{\delta} \tau_{r i g}^{d}=\boldsymbol{M}_{r i g \star}\left(\boldsymbol{q}_{\star}\right) \boldsymbol{\delta} \ddot{\boldsymbol{q}}+\boldsymbol{C}_{\star}\left(\boldsymbol{q}_{\star}, \dot{\boldsymbol{q}}_{\star}\right) \boldsymbol{\delta} \dot{\boldsymbol{q}}+\boldsymbol{F}_{v \star} \boldsymbol{\delta} \dot{\boldsymbol{q}}
\end{aligned}
$$

The term $\delta \tau^{p}$ can be rewritten in a linear form with respect to $\boldsymbol{\delta} \chi_{\text {rig }}$ using (10). A similar expression can also be derived for the elastic-joint case with the same method using the notations $f l_{1}$ and $f l_{2}$ respectively for the expressions (2) or (4) of the motion torque in the elastic-joint case. In the first case, we obtain

$$
\boldsymbol{\delta} \tau_{f l 1}=\boldsymbol{\delta} \tau_{f l 1}^{p}+\boldsymbol{\delta} \tau_{f l 1}^{d}
$$

with

$$
\begin{aligned}
\boldsymbol{\delta} \tau_{f l 1}^{p} & =\boldsymbol{\delta} \boldsymbol{M}(\boldsymbol{q}) \ddot{\boldsymbol{q}}+\boldsymbol{\delta} \boldsymbol{C}(\boldsymbol{q}, \dot{\boldsymbol{q}}) \dot{\boldsymbol{q}}+\boldsymbol{\delta} \boldsymbol{G}(\boldsymbol{q})+\boldsymbol{\delta} \boldsymbol{F}_{v a} \dot{\boldsymbol{q}} \\
& +\boldsymbol{\delta} \boldsymbol{K}(\boldsymbol{q}-\boldsymbol{\theta}) \\
\boldsymbol{\delta} \tau_{f l 1}^{d} & =\boldsymbol{M}_{\star}\left(\boldsymbol{q}_{\star}\right) \boldsymbol{\delta} \ddot{\boldsymbol{q}}+\boldsymbol{C}_{\star}\left(\boldsymbol{q}_{\star}, \dot{\boldsymbol{q}}_{\star}\right) \boldsymbol{\delta} \dot{\boldsymbol{q}}+\boldsymbol{F}_{v a \star} \boldsymbol{\delta} \dot{\boldsymbol{q}}
\end{aligned}
$$

In the second case, we obtain

$$
\boldsymbol{\delta} \tau_{f l 2}=\boldsymbol{\delta} \tau_{f l 2}^{p}+\boldsymbol{\delta} \tau_{f l 2}^{d}
$$

with

$$
\begin{aligned}
\boldsymbol{\delta} \tau_{f l 2}^{p} & =\boldsymbol{\delta} \boldsymbol{M}(\boldsymbol{q}) \ddot{\boldsymbol{q}}+\boldsymbol{\delta} \boldsymbol{C}(\boldsymbol{q}, \dot{\boldsymbol{q}}) \dot{\boldsymbol{q}}+\boldsymbol{\delta} \boldsymbol{G}(\boldsymbol{q})+\boldsymbol{\delta} \boldsymbol{F}_{v a} \dot{\boldsymbol{q}} \\
& +\boldsymbol{\delta} \boldsymbol{J}_{m o t} \ddot{\boldsymbol{\theta}}+\boldsymbol{\delta} \boldsymbol{F}_{v m} \dot{\boldsymbol{\theta}} \\
\boldsymbol{\delta} \boldsymbol{\tau}_{f l 2}^{d} & =\boldsymbol{M}_{\star}\left(\boldsymbol{q}_{\star}\right) \boldsymbol{\delta} \ddot{\boldsymbol{q}}+\boldsymbol{C}_{\star}\left(\boldsymbol{q}_{\star}, \dot{\boldsymbol{q}}_{\star}\right) \boldsymbol{\delta} \dot{\boldsymbol{q}}+\boldsymbol{F}_{v a \star} \boldsymbol{\delta} \dot{\boldsymbol{q}} \\
& +\boldsymbol{J}_{m o t \star} \boldsymbol{\delta} \ddot{\boldsymbol{\theta}}+\boldsymbol{F}_{v m \star} \boldsymbol{\delta} \dot{\boldsymbol{\theta}}
\end{aligned}
$$

Expressions (22) and (23) of the errors in both methods $f l_{1}$ and $f l_{2}$ allow to select the method that minimizes the effect of uncertainties, depending on which flexible parameters generate less error between stiffness or motor inertia and friction.

Finally, in both the rigid case (21) or in the elastic-joint case (22-23), the different expressions of $\delta \boldsymbol{\tau}$ are all composed of a term $\delta \tau^{p}$ due to parametric uncertainties and a term $\delta \tau^{d}$ due to numerical differentiation errors. Thus, these formulations allow to evaluate if the parameters estimation errors prevail or if the numerical differentiation errors are predominant (e.g. in case of trajectory with rapid changes). They also give a quantitative evaluation of the influence of uncertainties that can be expected online for a given trajectory.

\section{Bounding the estimation errors}

From the expression of uncertainties in both the rigid (21) and elastic-joint (22-23) cases, the uncertainties-induced errors on the detection method can be bounded. In the following, dependencies in motor and joint coordinates are removed in the notations for the sake of simplicity.

a) Errors $\delta \tau^{p}$ due to parametric uncertainties: It is assumed in II-B that $\delta \boldsymbol{\chi}$ is Gaussian such that $\delta \boldsymbol{\chi} \sim$ $\mathcal{N}\left(0, \sigma_{\chi_{\star}}^{2}\right)$ with $\boldsymbol{\sigma}_{\chi_{\star}}^{2}$ the diagonal covariance matrix of $\chi_{\star}$. Thus, the errors $\delta \tau^{p}$ are also Gaussian with $\delta \tau_{\text {rig }}^{p}=$ $\boldsymbol{\varphi}_{\text {rig }} \boldsymbol{\delta} \chi_{\text {rig }} \sim \mathcal{N}\left(0, \varphi_{\text {rig }} \boldsymbol{\sigma}_{\chi_{\text {rig }}}^{2} \boldsymbol{\varphi}_{\text {rig }}^{T}\right)$ in the rigid case, or $\boldsymbol{\delta} \tau_{f l}^{p}=\boldsymbol{\varphi}_{f l} \boldsymbol{\delta} \chi_{f l} \sim \mathcal{N}\left(0, \boldsymbol{\varphi}_{f l} \boldsymbol{\sigma}_{\chi_{f l \star}}^{2} \boldsymbol{\varphi}_{f l}^{T}\right)$ in the elasticjoint case. We denote $\sigma_{p}=\sqrt{\left(\varphi_{\text {rig }} \sigma_{\chi_{\text {rig }}}^{2} \varphi_{\text {rig }}^{T}\right) \text { or }}$ 
$\left.\sqrt{(} \boldsymbol{\varphi}_{f l} \boldsymbol{\sigma}_{\chi_{f l \star}}^{2} \boldsymbol{\varphi}_{f l}^{T}\right)$ the resulting standard deviation of $\delta \tau^{p}$ in both cases. Consequently, provided a reference trajectory along which $\varphi_{\text {rig }}$ or $\varphi_{f l}$ can be determined, for a given standard deviation $\sigma_{\chi_{\star}}$ of the parameters estimates, and in accordance with the normal law, confidence intervals can be computed for the errors $\delta \tau^{p}$ due to parametric uncertainties. For example, the probability that $\delta \tau^{p}$ belongs to the interval $\left[-3 \sigma_{p}, 3 \sigma_{p}\right]$ is $99,7 \%$.

b) Errors $\delta \tau^{d}$ due to numerical differentiation: In Section II-C, the errors due to the numerical differentiation were separated in two components, one due to the derivative approximation, and one due to filtered measurement noise. Let us first consider the term due to the derivative approximation in velocities estimates, corresponding to the transfer function $[H(s)-s]$ between the exact position input and its filtered derivative error output. For a given position trajectory characterized by velocities bounded by $v_{\max }$ and accelerations bounded by $a_{\max }$, the input trajectory can be approximated for analysis purposes by an equivalent sinusoidal signal of amplitude $E_{e q}=v_{\max }^{2} / a_{\max }$ and pulsation $\omega_{e q}=a_{\max } / v_{\max }$. Therefore the term due to the derivative approximation in velocities estimates can be bounded by $A_{d}=\left|H\left(j \omega_{e q}\right)-j \omega_{e q}\right| E_{e q}$. The term due to the derivative approximation in accelerations estimates is similarly bounded by $A_{d d}=\left|H\left(j \omega_{e q}\right)^{2}-j \omega_{e q}^{2}\right| E_{e q}$.

Let us now consider the term due to filtered measurement noise in velocities estimates for the $j^{t h}$ axis. In discrete time, if the input noise $\xi_{j}$ is white of variance $\sigma_{\xi_{j}}^{2}$ (e.g. quantization noise), the output noise filtered by $G(z)$ (of impulse response $g[k])$ is Gaussian of variance $\sigma_{d_{j}}^{2}=\sigma_{\xi_{j}}^{2} \sum_{k=-\infty}^{\infty} g^{2}[k]=$ $\sigma_{\xi_{j}}^{2} \int_{-1 / 2}^{1 / 2}|G(\nu)|^{2} d \nu$. For the two considered numerical differentiation method, we obtain

- Method $D_{1}$ :

$$
\sigma_{d_{j}}^{2}=\sigma_{\xi_{j}}^{2} 2\left(a^{2}+b^{2}\right) \text { with } a=\frac{2}{10 T_{s}}, b=\frac{1}{10 T_{s}}
$$

- Method $D_{2}$ :

$$
\sigma_{d_{j}}^{2}=\sigma_{\xi_{j}}^{2} \frac{2 \alpha^{2}}{1+\beta} \text { with } \alpha, \beta \text { defined in (12) }
$$

We proceed similarly for the accelerations estimates to find the output noise variance $\sigma_{d d_{j}}^{2}$. Similarly than in the previous analysis of $\delta \tau^{p}$, it is therefore possible, in accordance with the normal law, to define confidence intervals for the part of the errors $\delta \tau^{d}$ due to filtered noise. For the $99,7 \%$-confidence interval, the upper bound would be $3 \sigma_{d}$.

Finally, $\boldsymbol{\delta} \dot{\boldsymbol{q}}$ can be bounded by $\pm\left(\boldsymbol{A}_{\boldsymbol{d}}+3 \boldsymbol{\sigma}_{\boldsymbol{d}}\right)$ and $\boldsymbol{\delta} \ddot{\boldsymbol{q}}$ can be bounded by $\pm\left(\boldsymbol{A}_{\boldsymbol{d} \boldsymbol{d}}+3 \boldsymbol{\sigma}_{\boldsymbol{d} \boldsymbol{d}}\right)$. Thus, knowing the bounds on $\boldsymbol{\delta} \dot{\boldsymbol{q}}$ and $\boldsymbol{\delta} \ddot{\boldsymbol{q}}$, the expression of $\boldsymbol{\delta} \boldsymbol{\tau}^{d}$ detailed in (21) can be bounded for a given trajectory along which the matrices $M$ and $\boldsymbol{C}$ are evaluated.

\section{SIMULATION RESULTS}

The previously detailed results on the quantification of errors induced by parametric and numerical differentiation uncertainties were tested in simulation in the rigid case to compare their respective contributions to the error torque $\delta \boldsymbol{\tau}$ in different situations. The case study concerns a 6-DOF robot manipulator where motor positions are available from encoders on motor shafts. The structure of the robot is presented in Fig. 2.

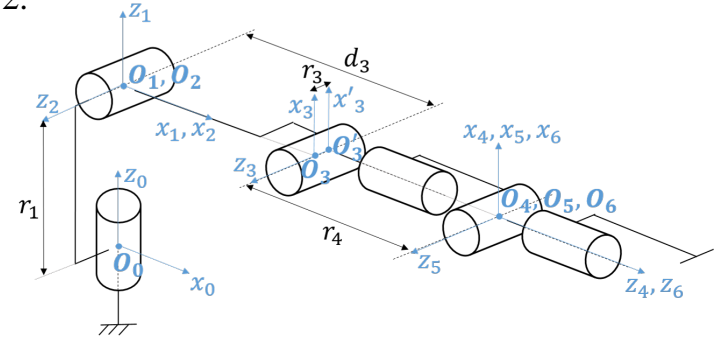

Fig. 2: Kinematics model of the serial robot manipulator with associated frames by Denavit-Hartenberg convention

For the simulations, each axis is controlled in position with a proportional-integral-derivative (PID) control law. The sample time is $1 \mathrm{~ms}$. The studied configurations are the combinations of $\left[T_{i}, U_{j}, D_{k}\right]$ with $i, j, k=1 . .2$ (see Table I). They are composed of trajectories with different dynamics (amplitude $E_{0}$, frequency $f_{0}$ ) and with varying accuracy in parameters estimation or numerical differentiation schemes. While velocities and accelerations of the sinusoidal trajectory will be sinusoidal, the triangular trajectory is more unfavorable since the acceleration peaks will excite the errors due to the uncertainties of the inertia matrix. We consider first favorable identification results where for each parameter $i$, $\% \boldsymbol{\sigma}_{\chi_{\star}, i} \in[-5 \%, 5 \%]$ and then an unfavorable case with ${ }_{0} \boldsymbol{\sigma}_{\chi_{\star, i}} \in[-20 \%, 20 \%]$. For this purpose, each $\%_{\boldsymbol{\chi}_{\star, i}}$ is fixed randomly and independently within these ranges. Noise on the position measurement is modeled by a white noise with zero-mean and variance equal to $\kappa^{2} / 12$ where $\kappa$ is the position

\begin{tabular}{|c|c|}
\hline \multirow{2}{*}{ Trajectory } & $\begin{array}{l}T_{1}: \text { Sinusoidal for the } 6 \text { axes } \\
\begin{aligned} E_{0} & =[0.2,0.2,0.2,0.2,0.2,0.2] \mathrm{rad} \\
f_{0} & =[0.7,0.6,0.5,0.4,0.3,0.2] \mathrm{Hz}\end{aligned}\end{array}$ \\
\hline & $\begin{array}{l}T_{2}: \text { Filtered triangular for the } 6 \text { axes } \\
\begin{aligned} E_{0} & =[0.2,0.2,0.2,0.2,0.2,0.2] \mathrm{rad} \\
f_{0} & =[0.7,0.6,0.5,0.4,0.3,0.2] \mathrm{Hz}\end{aligned}\end{array}$ \\
\hline \multirow{2}{*}{$\begin{array}{l}\% \sigma_{\chi_{\star}, i} \\
i=1 . . n_{b}\end{array}$} & $U_{1}:[-5 \%, 5 \%]$ \\
\hline & $U_{2}:[-20 \%, 20 \%]$ \\
\hline \multirow{2}{*}{$\begin{array}{l}\text { Numerical } \\
\text { differentiation } \\
\text { scheme }\end{array}$} & $\begin{array}{l}D_{1}: \text { Fourth-order backward finite difference } \\
\sigma_{d_{j}}^{2}=1.00 \cdot 10^{5} \sigma_{\xi_{j}}^{2} ; \sigma_{d d_{j}}^{2}=1.98 \cdot 10^{10} \sigma_{\xi_{j}}^{2}\end{array}$ \\
\hline & $\begin{array}{l}D_{2}: \text { First-order low-pass filtered derivative } \\
\quad \text { of cut-off frequency } \omega_{c}=2 \pi 110 \mathrm{rad} / \mathrm{s} \\
\sigma_{d_{j}}^{2}=3.55 \cdot 10^{5} \sigma_{\xi_{j}}^{2} ; \sigma_{d d_{j}}^{2}=1.48 \cdot 10^{11} \sigma_{\xi_{j}}^{2}\end{array}$ \\
\hline
\end{tabular}
sensor resolution.

TABLE I: Simulated configurations

From all combinations of $\left[T_{i}, U_{j}, D_{k}\right], i, j, k=1 . .2$, the maximum error between the exact expression (20) of $\delta \tau$ and the expression (21) due to the approximation explained in III-B is $\epsilon=10^{-2}[2.16 ; 1.66 ; 1.03 ; 0.87 ; 0.57 ; 0.34] \mathrm{Nm}$ which is negligible with respect to the applied motor torques. Results 
are presented for axis 1 which is the most inertial axis but the least subject to the effects of gravity and for axes 3 and 4 which are more affected by gravity-related uncertainties, with axis 4 belonging to the robot wrist.

First, the contribution of each type of uncertainties is examined without any collision. Simulation results on uncertaintiesinduced and numerical differentiation errors are presented in Fig. 3 for $T_{1}$ and in Fig. 4 for $T_{2}$. All the simulations are realized with the same sets of randomly and normally distributed parametric uncertainties according to $U_{1}$ or $U_{2}$. Results are presented for numerical differentiation scheme $D_{1}$ as it filters more high frequencies due to noise than $D_{2}$ for the chosen $\omega_{c}$ (see Fig. 1). For both $T_{1}$ and $T_{2}$ trajectories, the $\left[-3 \sigma_{p}, 3 \sigma_{p}\right]$-envelop and the $\left[\boldsymbol{\delta} \tau_{\text {rig,min }}^{d}, \boldsymbol{\delta} \tau_{\text {rig,max }}^{d}\right]$-envelop with $\boldsymbol{\delta} \dot{\boldsymbol{q}}$ bounded by $\pm\left(\boldsymbol{A}_{\boldsymbol{d}}+3 \boldsymbol{\sigma}_{\boldsymbol{d}}\right)$ and $\boldsymbol{\delta} \ddot{\boldsymbol{q}}$ bounded by $\pm\left(\boldsymbol{A}_{\boldsymbol{d d}}+3 \boldsymbol{\sigma}_{\boldsymbol{d d}}\right)$, both determined along the trajectory, represent the worst case of errors respectively due to parametric uncertainties and to numerical differentiation and give a reliable boundary of these errors. For favorable identification results (e.g. for the configurations $\left[T_{1}, U_{1}, D_{1}\right]$ or $\left[T_{2}, U_{1}, D_{1}\right]$ ), the errors due to model-uncertainties and numerical differentiation are of the same order of amplitude. However when model parameters are poorly identified (e.g. for the configurations $\left[T_{1}, U_{2}, D_{1}\right]$ or $\left.\left[T_{2}, U_{2}, D_{1}\right]\right)$, for each axis the error term due to model-uncertainties becomes dominant over the numerical differentiation error.
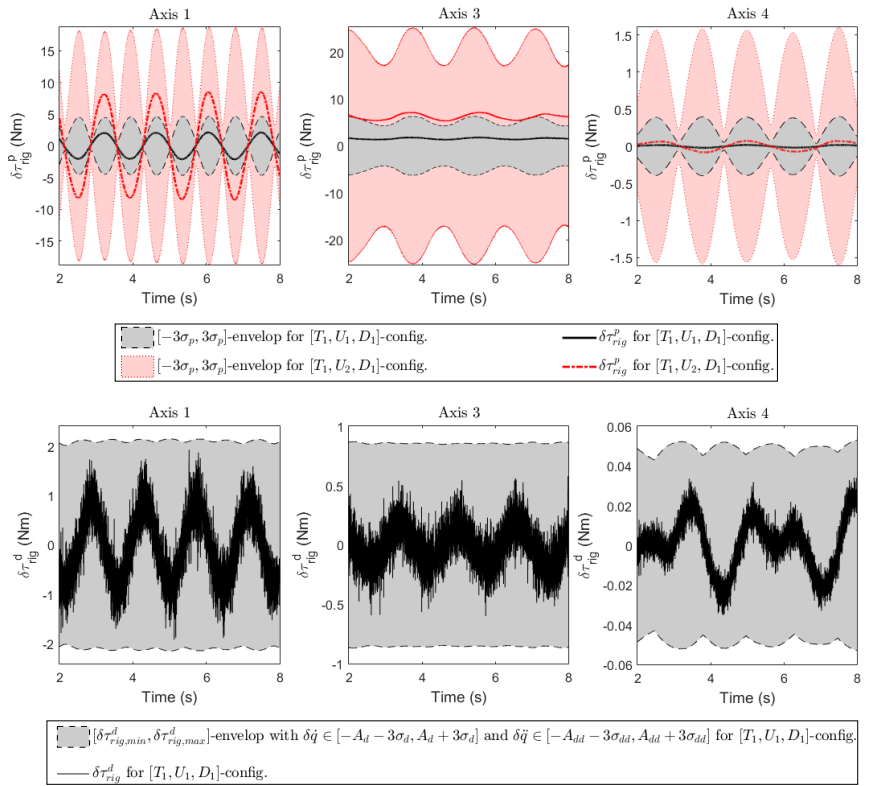

Fig. 3: Uncertainties-induced errors on trajectory $T_{1}$

The effect of uncertainties on the residual generation is then studied in presence of an impact for the configuration $\left[T_{1}, U_{1}, D_{1}\right]$. To simulate a collision, an external torque of $1 \mathrm{Nm}$ during 0,5 seconds is applied on each axis one after the other in order to emphasize the effects on each axis. Results are illustrated in Fig. 5. Parameters and numerical differentiation uncertainties significantly affect the residual which is not only the external torque as in the ideal case. Boundaries on $\delta \tau_{\text {rig }}$ are also represented on Fig. 5 as the sum

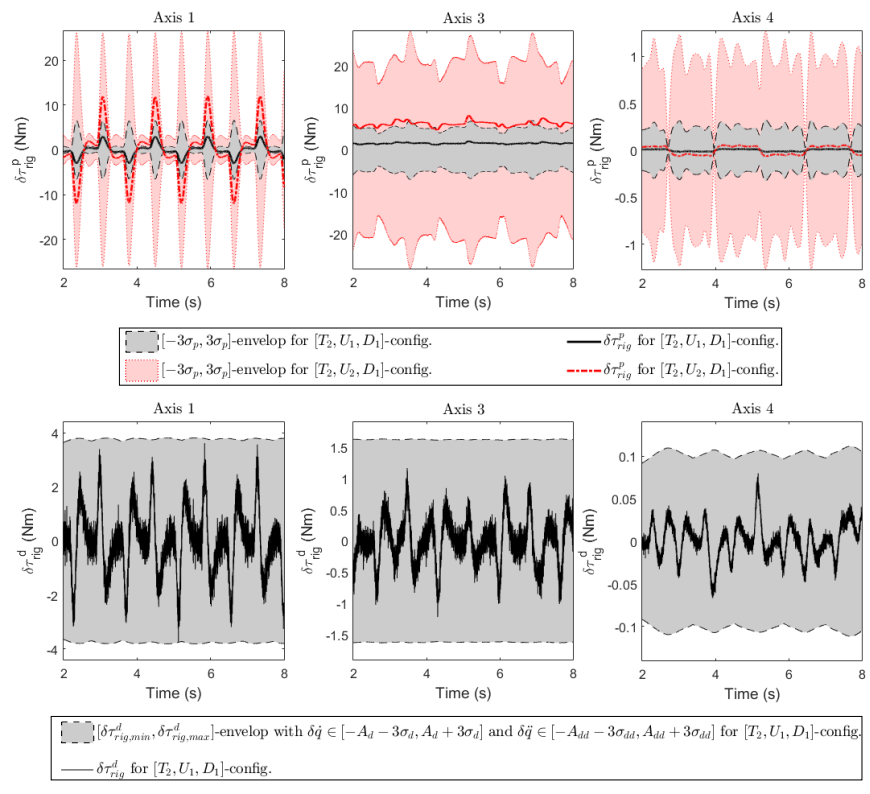

Fig. 4: Uncertainties-induced errors on trajectory $T_{2}$

of the $\left[-3 \sigma_{p}, 3 \sigma_{p}\right]$-envelop and the $\left[\delta \tau_{\text {rig,min }}^{d}, \delta \tau_{\text {rig,max }}^{d}\right]$ envelop with $\delta \dot{q}$ bounded by $\pm\left(A_{d}+3 \sigma_{d}\right)$ and $\delta \ddot{q}$ bounded by $\pm\left(\boldsymbol{A}_{\boldsymbol{d} \boldsymbol{d}}+3 \boldsymbol{\sigma}_{\boldsymbol{d d}}\right)$, where both envelops are determined for the configuration $\left[T_{1}, U_{1}, D_{1}\right]$ along the trajectory with collision. For axes 1 and 3, given that the boundaries on the uncertainties-induced errors are above $1 \mathrm{Nm}$ at the moment of the collision, the impact can not be detected on these axes, while on axis 4 the boundaries are smaller than the amplitude of the collision, which means that the detection method is sensitive enough to detect the collision. Thus simulating these boundaries allows to predict the minimum amplitude of the collision that can be detected, depending on the accuracy of the parameter estimation and the selected numerical differentiation scheme. The sensitivity of the detection can be increased by reducing the width of the envelopes (e.g. considering $\left[-2 \sigma_{p}, 2 \sigma_{p}\right]$ instead of $\left.\left[-3 \sigma_{p}, 3 \sigma_{p}\right]\right)$ but at the expense of increasing the risk of false alarms due to uncertainties.
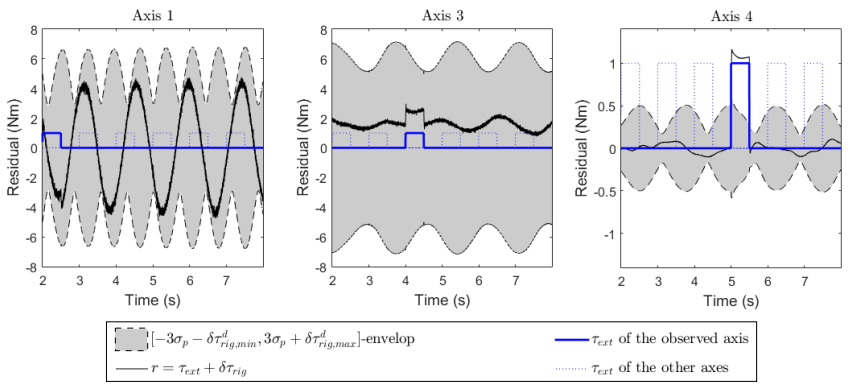

Fig. 5: Residual generated in presence of a collision on each axis separately with the configuration $\left[T_{1}, U_{1}, D_{1}\right]$

\section{CONCLUSION AND PERSPECTIVES}

In this paper, an evaluation of the errors due to uncertainties has been derived for a basic collision detection method using the inverse dynamic model of both rigid and elasticjoint robots. These uncertainties-induced errors have been 
decomposed into a combination of parameters estimation and numerical differentiation errors and a method to obtain their boundaries has been proposed. This approach allows to quantify the influence of the uncertainties on the collision detection, to evaluate which uncertainties source will generate more errors and to determine in simulation the expected sensitivity of the collision detection method depending on the uncertainties.

This method can be extended to other model-based collision detection approaches (e.g. collision detection methods based on the generalized momentum) in order to compare their performance in presence of uncertainties in a quantified way and determine the favorable cases for each method.

\section{REFERENCES}

[1] S. Haddadin, A. Albu-Schäffer, and G. Hirzinger, "Safety evaluation of physical human-robot interaction via crash-testing." in Robotics: Science and Systems, vol. 3, 2007, pp. 217-224.

[2] M. Vasic and A. Billard, "Safety issues in human-robot interactions," in IEEE Int. Conference on Robotics and Automation (ICRA), 2013, May 2013, pp. 197-204.

[3] E. Martinez-Martin and A. P. del Pobil, "Visual people detection for safe human-robot interaction," in IEEE RO-MAN, 2013, Aug 2013, pp. 326-327.

[4] S. Phan, Z. F. Quek, P. Shah, D. Shin, Z. Ahmed, O. Khatib, and M. Cutkosky, "Capacitive skin sensors for robot impact monitoring," in IEEE/RSJ Int. Conference on Intelligent Robots and Systems (IROS), 2011, Sept 2011, pp. 2992-2997.

[5] A. Cirillo, P. Cirillo, G. D. Maria, C. Natale, and S. Pirozzi, "A proximity/contact-force sensor for human safety in industrial robot environment," in IEEE/ASME Int. Conference on Advanced Intelligent Mechatronics (AIM), 2013, July 2013, pp. 1272-1277.

[6] S.-D. Lee, Y.-L. Kim, and J.-B. Song, "Novel collision detection index based on joint torque sensors for a redundant manipulator," in IEEE/RSJ Int. Conference on Intelligent Robots and Systems (IROS), 2013, Nov 2013, pp. 4636-4641.

[7] V. Venkatasubramanian, R. Rengaswamy, K. Yin, and S. N. Kavuri, "A review of process fault detection and diagnosis: Part I: Quantitative model-based methods," Computers \& chemical engineering, vol. 27, no. 3, pp. 293-311, 2003.

[8] S. Morinaga and K. Kosuge, "Collision detection system for manipulator based on adaptive impedance control law," in IEEE Int. Conference on Robotics and Automation (ICRA), 2003, vol. 1, Sept 2003, pp. 10801085 vol.1.

[9] M. Geravand, F. Flacco, and A. D. Luca, "Human-robot physical interaction and collaboration using an industrial robot with a closed control architecture," in IEEE Int. Conference on Robotics and Automation (ICRA), 2013, May 2013, pp. 4000-4007.

[10] A. D. Luca, A. Albu-Schaffer, S. Haddadin, and G. Hirzinger, "Collision detection and safe reaction with the DLR-III lightweight manipulator arm," in IEEE/RSJ Int. Conference on Intelligent Robots and Systems (IROS), 2006, Oct 2006, pp. 1623-1630.

[11] S. Haddadin, A. Albu-Schaffer, A. D. Luca, and G. Hirzinger, "Collision detection and reaction: A contribution to safe physical human-robot interaction," in IEEE/RSJ Int. Conference on Intelligent Robots and Systems (IROS), 2008, Sept 2008, pp. 3356-3363.

[12] A. Wahrburg, E. Morara, G. Cesari, B. Matthias, and H. Ding, "Cartesian contact force estimation for robotic manipulators using kalman filters and the generalized momentum," in IEEE Int. Conference on Automation Science and Engineering (CASE), 2015, Aug 2015, pp. 1230-1235.

[13] A. De Luca and R. Mattone, "An adapt-and-detect actuator FDI scheme for robot manipulators," in IEEE Int. Conference on Robotics and Automation (ICRA), 2004, vol. 5, 2004, pp. 4975-4980.

[14] M. Makarov, A. Caldas, M. Grossard, P. Rodriguez-Ayerbe, and D. Dumur, "Adaptive filtering for robust proprioceptive robot impact detection under model uncertainties," IEEE/ASME Transactions on Mechatronics, vol. 19, no. 6, pp. 1917-1928, Dec 2014.

[15] W. Khalil and E. Dombre, Modeling, identification and control of robots. Butterworth-Heinemann, 2004.
[16] C. Lightcap and S. Banks, "Dynamic identification of a mitsubishi pa10-6ce robot using motion capture," in IEEE/RSJ Int. Conference on Intelligent Robots and Systems (IROS), 2007, Oct 2007, pp. 3860-3865. 